\title{
Stability of Highly Nonlinear Neutral Stochastic Differential Delay Equations
}

\author{
Mingxuan Shen, Weiyin Fei, Xuerong Mao, Yong Liang
}

\begin{abstract}
Stability criteria for neutral stochastic differential delay equations (NSDDEs) have been studied intensively for the past several decades. Most of these criteria can only be applied to NSDDEs where their coefficients are either linear or nonlinear but bounded by linear functions. This paper is concerned with the stability of hybrid NSDDEs without the linear growth condition, to which we will refer as highly nonlinear ones. The stability criteria established in this paper will be dependent on delays.
\end{abstract}

Index Terms-Neutral stochastic differential delay equation; Nonlinear growth condition; Asymptotic stability; Delay dependent; Markovian switching

\section{INTRODUCTION}

Many stochastic dynamical systems do not only depend on present and past states but also involve derivatives with delays. Neutral stochastic differential delay equations (NSDDEs) are often used to model such systems. NSDDEs with Markovian switching (also known as hybrid NSDDEs) form an important class of hybrid dynamical systems. They have been successfully applied in practice, such as in traffic control, switching power converters, neural networks, and so on (see, e.g., [1-5]). The research on the stability of NSDDEs with Markovian switching has received considerable attention for the past several decades (see, e.g., [6-9]). The stability criteria are in general classified into two categories: delay-dependent and delayindependent stability criteria. The delay-dependent stability criteria take into account the size of delays and hence are generally less conservative than the delay-independent ones which work for any size of delays.

A common feature of the existing delay-dependent stability criteria is that most of them can only be applied to delay systems where their coefficients are either linear or nonlinear but bounded by linear functions (see, e.g., [10-12]). However, the linear growth condition is usually violated in many practical applications. Recently, there are some progress on stability for highly nonlinear stochastic delay systems. For example, the stability and boundedness of nonlinear hybrid SDDEs were studied in [13], the robust stability and boundedness of SDDEs without the linear growth condition were studied in [14], the stability of neutral stochastic differential equations with unbounded delay and Markovian switching was studied in [15]. But those results are all delay independent. [16] is the first to establish delay-dependent criteria for highly nonlinear hybrid SDDEs . However, to the best of our knowledge, there is so far no delay-dependent stability criteria for highly nonlinear hybrid NSDDEs. Motivated by [16], the key

M. Shen is with the School of Science, Nanjing University of Science and Technology, Nanjing, Jiangsu 210094, China and also with School of Mathematics and Physics, Anhui Polytechnic University, Wuhu, Anhui 241000, China.smx1011@163.com

W. Fei is with the School of Mathematics and Physics, Anhui Polytechnic University, Wuhu, Anhui 241000, China. Corresponding author. wyfei@ahpu.edu.cn.

$\mathrm{X}$. Mao is with the Department of Mathematics and Statistics, University of Strathclyde, Glasgow G1 1XH, UK. x.mao@strath.ac.uk.

Y. Liang is with the School of Mathematics, Shanghai University of Finance and Economics, Shanghai, 200433, China and also with School of Mathematics and Physics, Anhui Polytechnic University, Wuhu, Anhui 241000, China. liangyong@ahpu.edu.cn. aim of this paper is to establish the delay-dependent stability criteria for hybrid NSDDEs with the polynomial growth condition instead of the linear growth condition. To explain our aim more clearly, let us consider the scalar highly nonlinear hybrid NSDDE

$$
\begin{aligned}
d[x(t)-D(x(t-\tau))]= & f(x(t), x(t-\tau), r(t), t) d t \\
& +g(x(t), x(t-\tau), r(t), t) d B(t),
\end{aligned}
$$

where $x(t) \in \mathbb{R}$ is the state, $\tau$ stands for time delay, $B(t)$ is a scalar Brownian motion, $r(t)$ is a Markov chain on the state space $\mathbb{S}=\{1,2\}$ with its generator

$$
\Gamma=\left(\begin{array}{cc}
-1 & 1 \\
2 & -2
\end{array}\right)
$$

and the coefficients are defined by

$$
\begin{aligned}
& f(x, y, 1, t)=-y-4 x^{3}, \quad f(x, y, 2, t)=-y-5 x^{3}, \\
& g(x, y, 1, t)=g(x, y, 2, t)=0.5 y^{2}, \\
& D(x(t-\tau))=0.1 x(t-\tau) .
\end{aligned}
$$

This nonlinear hybrid NSDDE can be regarded as that it operates in two modes and it obeys

$$
\begin{aligned}
d[x(t)-0.1 x(t-\tau)]= & {\left[-x(t-\tau)-4 x^{3}(t)\right] d t } \\
& +0.5 x^{2}(t-\tau) d B(t), \\
d[x(t)-0.1 x(t-\tau)]= & {\left[-x(t-\tau)-5 x^{3}(t)\right] d t } \\
& +0.5 x^{2}(t-\tau) d B(t)
\end{aligned}
$$

in mode 1 and 2, respectively. The system will switch from one mode to the other according to the probability law of the Markov chain. If $\tau=0.01$, the computer simulation shows that the hybrid NSDDE is asymptotically stable. If the time-delay is large, say $\tau=2$, the computer simulation shows that the hybrid NSDDE is unstable. In other words, whether the hybrid NSDDE is stable or not depends on how small or large the time-delay is. On the other hand, both drift and diffusion coefficients of the hybrid NSDDE are highly nonlinear. Unfortunately, there is so far no delay-dependent criterion which can be applied to this NSDDE to derive a sufficient bound on the timedelay $\tau$ for the NSDDE to be stable. Our aim here is to establish delay-dependent criteria for such highly nonlinear hybrid NSDDEs.

\section{NOTATION AND ASSUMPTION}

Throughout this paper, unless otherwise specified, we use the following notation. If $A$ is a vector or matrix, its transpose is denoted by $A^{T}$. If $x \in \mathbb{R}^{n}$, then $|x|$ is its Euclidean norm. For a matrix $A$, it's trace norm is denoted by $|A|=\sqrt{\operatorname{trace}\left(A^{T} A\right)}$. For $\tau>0$, denote by $C\left([-\tau, 0] ; \mathbb{R}^{n}\right)$ the family of continuous functions $\varphi$ from $[-\tau, 0] \rightarrow \mathbb{R}^{n}$ with the norm $\|\varphi\|=\sup _{-\tau \leq u \leq 0}|\varphi(u)|$. Let $\left(\Omega, \mathcal{F},\left\{\mathcal{F}_{t}\right\}_{t \geq 0}, \mathbb{P}\right)$ be a complete probability space with a filtration $\left\{\mathcal{F}_{t}\right\}_{t \geq 0}$ satisfying the usual conditions (i.e. it is increasing and right continuous while $\mathcal{F}_{0}$ contains all $\mathbb{P}$-null sets). Let $B(t)=\left(B_{1}(t), \cdots, B_{m}(t)\right)^{T}$ be an $m$-dimensional Brownian motion defined on the probability space. Let $r(t), t \geq 0$, be a rightcontinuous Markov chain on the probability space taking values in a finite state space $\mathbb{S}=\{1,2, \cdots, N\}$ with generator $\Gamma=\left(\gamma_{i j}\right)_{N \times N}$, 
Here $\gamma_{i j} \geq 0$ is the transition rate from $i$ to $j$ if $i \neq j$ while $\gamma_{i i}=-\sum_{j \neq i} \gamma_{i j}$. We assume that the Markov chain $r(\cdot)$ is independent of the Brownian motion $B(\cdot)$. Let

$$
\begin{aligned}
& f: \mathbb{R}^{n} \times \mathbb{R}^{n} \times \mathbb{S} \times \mathbb{R}_{+} \rightarrow \mathbb{R}^{n}, \\
& g: \mathbb{R}^{n} \times \mathbb{R}^{n} \times \mathbb{S} \times \mathbb{R}_{+} \rightarrow \mathbb{R}^{n \times m}, \quad D: \mathbb{R}^{n} \rightarrow \mathbb{R}^{n}
\end{aligned}
$$

be Borel measurable functions. Consider an $n$-dimensional hybrid NSDDE

$$
\begin{gathered}
d[x(t)-D(x(t-\tau))]=f(x(t), x(t-\tau), r(t), t) d t \\
+g(x(t), x(t-\tau), r(t), t) d B(t)
\end{gathered}
$$

on $t \geq 0$ with initial data

$$
\{x(t):-\tau \leq t \leq 0\}=\eta \in C\left([-\tau, 0] ; \mathbb{R}^{n}\right), r(0)=i_{0} \in \mathbb{S} .
$$

The well-known conditions imposed for the existence and uniqueness of the global solution are the local Lipschitz condition and the linear growth condition (see, e.g., [4, 9, 17]). In this paper, we need the local Lipschitz condition. However, we impose the polynomial growth condition instead of the linear growth condition. Let us state these conditions as an assumption for the use of this paper.

Assumption 2.1. Assume that for any $h>0$, there exists a positive constant $K_{h}$ such that

$$
\begin{aligned}
|f(x, y, i, t)-f(\bar{x}, \bar{y}, i, t)| & \vee|g(x, y, i, t)-g(\bar{x}, \bar{y}, i, t)| \\
& \leq K_{h}(|x-\bar{x}|+|y-\bar{y}|)
\end{aligned}
$$

for all $x, y, \bar{x}, \bar{y} \in \mathbb{R}^{n}$ with $|x| \vee|y| \vee|\bar{x}| \vee|\bar{y}| \leq h$ and all $(i, t) \in$ $\mathbb{S} \times \mathbb{R}_{+}$. Assume also that there exist three constants $K>0, q_{1} \geq 1$ and $q_{2} \geq 1$ such that

$$
\begin{aligned}
|f(x, y, i, t)| & \leq K\left(1+|x|^{q_{1}}+|y|^{q_{1}}\right), \\
|g(x, y, i, t)| & \leq K\left(1+|x|^{q_{2}}+|y|^{q_{2}}\right)
\end{aligned}
$$

for all $(x, y, i, t) \in \mathbb{R}^{n} \times \mathbb{R}^{n} \times \mathbb{S} \times \mathbb{R}_{+}$. Assume moreover that there is a constant $\kappa \in\left(0, \frac{\sqrt{2}}{2}\right)$ such that

$$
|D(u)-D(v)| \leq \kappa|u-v|
$$

for all $u, v \in \mathbb{R}$, and $D(0)=0$.

Of course, if $q_{1}=q_{2}=1$, then condition (2.4) is the familiar linear growth condition. However, we emphasise once again that we are here interested in highly nonlinear NSDDEs which have either $q_{1}>1$ or $q_{2}>1$. We will refer to condition (2.4) as the polynomial growth condition. Of course, without the linear growth condition, the solution of the NSDDE (2.1) may explode to infinity at a finite time. To avoid such a possible explosion, we need to impose an additional condition in terms of Lyapunov functions. For this purpose, we need more notation. Let $C^{2,1}\left(\mathbb{R}^{n} \times \mathbb{S} \times \mathbb{R}_{+} ; \mathbb{R}_{+}\right)$denote the family of non-negative functions $U(x, i, t)$ defined on $(x, i, t) \in \mathbb{R}^{n} \times \mathbb{S} \times \mathbb{R}_{+}$ which are continuously twice differentiable in $x$ and once in $t$. We now state another assumption.

Assumption 2.2. Assume that there exists a pair of functions $\bar{U} \in$ $C^{2,1}\left(\mathbb{R}^{n} \times \mathbb{S} \times \mathbb{R}_{+} ; \mathbb{R}_{+}\right)$and $G \in C\left(\mathbb{R}^{n} \times[-\tau, \infty) ; \mathbb{R}_{+}\right)$, as well as positive numbers $c_{1}, c_{2}, c_{3}$ and $q \geq 2\left(q_{1} \vee q_{2}\right)$, such that

$$
c_{3}<c_{2}, \quad|x|^{q} \leq \bar{U}(x, i, t) \leq G(x, t),
$$

$$
\begin{aligned}
\forall(x, i, t) & \in \mathbb{R}^{n} \times \mathbb{S} \times \mathbb{R}_{+}, \text {and } \\
\mathbb{L} \bar{U} & (x-D(y), y, i, t): \\
& =\bar{U}_{t}(x-D(y), i, t)+\bar{U}_{x}(x-D(y), i, t) f(x, y, i, t) \\
& +\frac{1}{2} \operatorname{trace}\left[g^{T}(x, y, i, t) \bar{U}_{x x}(x-D(y), i, t) g(x, y, i, t)\right] \\
& +\sum_{j=1}^{N} \gamma_{i j} \bar{U}(x-D(y), j, t) \\
& \leq c_{1}-c_{2} G(x, t)+c_{3} G(y, t-\tau),
\end{aligned}
$$

$\forall(x, y, i, t) \in \mathbb{R}^{n} \times \mathbb{R}^{n} \times \mathbb{S} \times \mathbb{R}_{+}$.

We now cite a result from [15] as a lemma for the use of this paper.

Lemma 2.3. Under Assumptions 2.1 and 2.2, the NSDDE (2.1) with the initial data (2.2) has the unique global solution $x(t)$ on $t \geq-\tau$ and the solution has the property that $\sup _{-\tau \leq t<\infty} \mathbb{E}|x(t)|^{q}<\infty$.

\section{Delay-Dependent Asymptotic Stability}

In this section, we will use the method of Lyapunov functionals to investigate the delay-dependent asymptotic stability. We define two segments $\bar{x}_{t}:=\{x(t+s):-2 \tau \leq s \leq 0\}$ and $\bar{r}_{t}:=\{r(t+s)$ : $-2 \tau \leq s \leq 0\}$ for $t \geq 0$. For $\bar{x}_{t}$ and $\bar{r}_{t}$ to be well defined for $0 \leq t<2 \tau$, we set $x(s)=\eta(-\tau)$ for $s \in[-2 \tau,-\tau)$ and $r(s)=r_{0}$ for $s \in[-2 \tau, 0)$. The Lyapunov functional used in this paper was defined by

$$
\begin{aligned}
V\left(\bar{x}_{t}, \bar{r}_{t}, t\right) & =U(x(t)-D(x(t-\tau)), r(t), t) \\
& +\theta \int_{-\tau}^{0} \int_{t+s}^{t}\left[\tau|f(x(v), x(v-\tau), r(v), v)|^{2}\right. \\
& \left.+|g(x(v), x(v-\tau), r(v), v)|^{2}\right] d v d s
\end{aligned}
$$

for $t \geq 0$, where $U \in C^{2,1}\left(\mathbb{R}^{n} \times \mathbb{S} \times \mathbb{R}_{+} ; \mathbb{R}_{+}\right)$such that

$$
\lim _{|x| \rightarrow \infty}\left[\inf _{(t, i) \in \mathbb{R}_{+} \times \mathbb{S}} U(x, r, t)\right]=\infty,
$$

and $\theta$ is a positive number to be determined later while we set

$$
f(x, y, i, s)=f(x, y, i, 0), \quad g(x, y, i, s)=g(x, y, i, 0)
$$

for $(x, y, i, s) \in \mathbb{R}^{n} \times \mathbb{R}^{n} \times \mathbb{S} \times[-2 \tau, 0)$. Applying the generalized Itô formula (see, e.g.,[5, Theorem 1.45 on page 48]) to $U(x(t), r(t), t)$, we get

$$
\begin{aligned}
& d U(x(t)-D(x(t-\tau)), r(t), t)) \\
& =\left(U_{t}(x(t)-D(x(t-\tau)), r(t), t)\right. \\
& +U_{x}(x(t)-D(x(t-\tau)), r(t), t) f(x(t), x(t-\tau), r(t), t) \\
& +\frac{1}{2} \operatorname{trace}\left[g^{T}(x(t), x(t-\tau), r(t), t)\right. \\
& \left.\quad \times U_{x x}(x(t)-D(x(t-\tau)), r(t), t) g(x(t), x(t-\tau), r(t), t)\right] \\
& \left.+\sum_{j=1}^{N} \gamma_{r(t), j} U(x(t)-D(x(t-\tau)), r(t), t)\right) d t+d M(t),
\end{aligned}
$$

for $t \geq 0$, where $M(t)$ is a continuous local martingale with $M(0)=$ 0 (see, e.g.,[5, Theorem 1.45 on page 48]). Rearranging terms gives

$$
\begin{aligned}
& d U(x(t)-D(x(t-\tau)), r(t), t) \\
& =\left(U_{x}(x(t)-D(x(t-\tau)), r(t), t)\right. \\
& \times[f(x(t), x(t-\tau), r(t), t)-f(x(t), x(t), r(t), t)] \\
& +\mathcal{L} U(x(t)-D(x(t-\tau)), x(t-\tau), r(t), t))+d M(t),
\end{aligned}
$$


where the function $\mathcal{L} U: \mathbb{R}^{n} \times \mathbb{R}^{n} \times \mathbb{S} \times \mathbb{R}_{+} \rightarrow \mathbb{R}$ is defined by

$$
\begin{aligned}
\mathcal{L} U & (x-D(y), y, i, t) \\
& =U_{t}(x-D(y), i, t)+U_{x}(x-D(y), i, t) f(x, x, i, t) \\
& +\frac{1}{2} \operatorname{trace}\left[g^{T}(x, y, i, t) U_{x x}(x-D(y), i, t) g(x, y, i, t)\right] \\
& +\sum_{j=1}^{N} \gamma_{i j} U(x-D(y), j, t) .
\end{aligned}
$$

Lemma 3.1. With the notation above, $V\left(\bar{x}_{t}, \bar{r}_{t}, t\right)$ is an Itô process on $t \geq 0$ with its Itô differential

$$
d V\left(\bar{x}_{t}, \bar{r}_{t}, t\right)=L V\left(\bar{x}_{t}, \bar{r}_{t}, t\right) d t+d M(t),
$$

where $M(t)$ is a continuous local martingale with $M(0)=0$ and

$$
\begin{aligned}
& L V\left(\bar{x}_{t}, \bar{r}_{t}, t\right)=U_{x}(x(t)-D(x(t-\tau)), r(t), t) \\
& \times[f(x(t), x(t-\tau), r(t), t)-f(x(t), x(t), r(t), t)] \\
& +\mathcal{L} U(x(t)-D(x(t-\tau)), x(t-\tau), r(t), t) \\
& +\theta \tau\left[\tau|f(x(t), x(t-\tau), r(t), t)|^{2}+|g(x(t), x(t-\tau), r(t), t)|^{2}\right] \\
& -\theta \int_{t-\tau}^{t}\left[\tau|f(x(v), x(v-\tau), r(v), v)|^{2}\right. \\
& \left.+|g(x(v), x(v-\tau), r(v), v)|^{2}\right] d v
\end{aligned}
$$

To study the delay-dependent asymptotic stability of the NSDDE (2.1), we need to impose a couple of new assumptions.

Assumption 3.2. Assume that there are functions $U \in C^{2,1}\left(\mathbb{R}^{n} \times\right.$ $\left.\mathbb{S} \times \mathbb{R}_{+} ; \mathbb{R}_{+}\right), U_{1} \in C\left(\mathbb{R}^{n} \times[-\tau, \infty) ; \mathbb{R}_{+}\right), W \in C\left(\mathbb{R}^{n} ; \mathbb{R}_{+}\right)$, and positive numbers $\alpha_{k}(k=1,2)$ and $\beta_{j}(j=1,2,3)$ such that

$$
\alpha_{2}<\alpha_{1}
$$

and

$$
\begin{aligned}
& \mathcal{L} U(x-D(y), y, i, t)+\beta_{1}\left|U_{x}(x-D(y), i, t)\right|^{2} \\
& +\beta_{2}|f(x, y, i, t)|^{2}+\beta_{3}|g(x, y, i, t)|^{2} \\
& \leq-\alpha_{1} U_{1}(x, t)+\alpha_{2} U_{1}(y, t-\tau)-W(x-D(y)),
\end{aligned}
$$

for all $(x, y, i, t) \in \mathbb{R}^{n} \times \mathbb{R}^{n} \times \mathbb{S} \times \mathbb{R}_{+}$. Furthermore $W$ has the property

$$
W(x)=0 \quad \text { if and only if } x=0 .
$$

Assumption 3.3. Assume that there exists a positive number $\beta_{4}$ such that

$$
|f(x, x, i, t)-f(x, y, i, t)| \leq \beta_{4}|x-y|
$$

for all $(x, y, i, t) \in \mathbb{R}^{n} \times \mathbb{R}^{n} \times \mathbb{S} \times \mathbb{R}_{+}$.

We can see (3.4) also implies

$$
\begin{aligned}
\mathcal{L} U(x & -D(y), y, i, t) \\
& \leq-\alpha_{1} U_{1}(x, t)+\alpha_{2} U_{1}(y, t-\tau)-W(x-D(y)) .
\end{aligned}
$$

Rearranging hybrid NSDDE (2.1) as

$$
\begin{aligned}
& d[x(t)-D(x(t-\tau))] \\
& =f(x(t), x(t), r(t), t) d t+g(x(t), x(t-\tau), r(t), t) d B(t) \\
& +[f(x(t), x(t-\tau), r(t), t)-f(x(t), x(t), r(t), t)] d t,
\end{aligned}
$$

we see that NSDDE (2.1) is a perturbed system of the stable NSDDE

$$
\begin{aligned}
& d[X(t)-D(X(t-\tau))] \\
& =f(X(t), X(t), r(t), t) d t+g(X(t), X(t-\tau), r(t), t) d B(t) .
\end{aligned}
$$

If the time delay is not too large, then the difference $f(x(t), x(t-$ $\tau), r(t), t)-f(x(t), x(t), r(t), t)$ would be small so that $x(t)$ should be close to $X(t)$.
Theorem 3.4. Let Assumptions 2.1, 2.2, 3.2 and 3.3 hold. Assume also that

$$
\tau \leq \frac{\left(1-2 \kappa^{2}\right) \beta_{1} \beta_{3}}{\beta_{4}^{2}} \wedge \frac{\sqrt{\left(1-2 \kappa^{2}\right) \beta_{1} \beta_{2}}}{\beta_{4}} .
$$

Then for any given initial data (2.2), the solution of the NSDDE (2.1) has the properties that

$$
\begin{gathered}
\int_{0}^{\infty} \mathbb{E} U_{1}(x(t), t) d t<\infty, \\
\sup _{0 \leq t<\infty} \mathbb{E} U(x(t)-D(x(t-\tau)), r(t), t)<\infty .
\end{gathered}
$$

Proof: Fix the initial data $\eta \in C\left([-\tau, 0] ; \mathbb{R}^{n}\right)$ and $r_{0} \in \mathbb{S}$ arbitrarily. Let $k_{0}>0$ be a sufficiently large integer such that $\|\eta\|:=\sup _{-\tau<s \leq 0} \eta(s)<k_{0}$. For each integer $k>k_{0}$, define the stopping time

$$
\sigma_{k}=\inf \{t \geq 0:|x(t)-D(x(t-\tau))| \geq k\},
$$

where throughout this paper we set $\inf \emptyset=\infty$ (as usual $\emptyset$ denotes the empty set). It is easy to see that $\sigma_{k}$ is increasing as $k \rightarrow \infty$ and $\lim _{k \rightarrow \infty} \sigma_{k}=\infty$ a.s. By the generalized Itô formula we obtain from Lemma 3.1 that

$$
\begin{aligned}
& \mathbb{E} V\left(\bar{x}_{t \wedge \sigma_{k}}, \bar{r}_{t \wedge \sigma_{k}}, t \wedge \sigma_{k}\right) \\
& \quad=V\left(\bar{x}_{0}, \bar{r}_{0}, 0\right)+\mathbb{E} \int_{0}^{t \wedge \sigma_{k}} L V\left(\bar{x}_{s}, \bar{r}_{s}, s\right) d s
\end{aligned}
$$

for any $t \geq 0$ and $k \geq k_{0}$. Let $\theta=\beta_{4}^{2} /\left(\beta_{1}\left(1-2 \kappa^{2}\right)\right)$. By Assumption 3.2 , it is easy to see that

$$
\begin{aligned}
& U_{x}(x(t)-D(x(t-\tau)), r(t), t) \\
& \quad \times[f(x(t), x(t-\tau), r(t), t)-f(x(t), x(t), r(t), t)] \\
& \leq \beta_{1}\left|U_{x}(x(t)-D(x(t-\tau)), r(t), t)\right|^{2}+\frac{\beta_{4}^{2}}{4 \beta_{1}}|x(t)-x(t-\tau)|^{2} .
\end{aligned}
$$

By condition (3.7), we also have

$$
\theta \tau^{2} \leq \beta_{2} \text { and } \theta \tau \leq \beta_{3} .
$$

It then follows from Lemma 3.1 that

$$
\begin{aligned}
& L V\left(\bar{x}_{s}, \bar{r}_{s}, s\right) \leq \mathcal{L} U(x(s)-D(x(s-\tau)), x(s-\tau), r(s), s) \\
& +\beta_{1}\left|U_{x}(x(s)-D(x(s-\tau)), r(s), s)\right|^{2} \\
& +\beta_{2}|f(x(s), x(s-\tau), r(s), s)|^{2} \\
& +\beta_{3}|g(x(s), x(s-\tau), r(s), s)|^{2}+\frac{\beta_{4}^{2}}{4 \beta_{1}}|x(s)-x(s-\tau)|^{2} \\
& \quad-\frac{\beta_{4}^{2}}{\beta_{1}\left(1-2 \kappa^{2}\right)} \int_{s-\tau}^{s}\left[\tau|f(x(v), x(v-\tau), r(v), v)|^{2}\right. \\
& \left.\quad+|g(x(v), x(v-\tau), r(v), v)|^{2}\right] d v .
\end{aligned}
$$

By Assumption 3.2, we then have

$$
\begin{gathered}
L V\left(\bar{x}_{s}, \bar{r}_{s}, s\right) \leq-\alpha_{1} U_{1}(x(s), s)+\alpha_{2} U_{1}(x(s-\tau), s-\tau) \\
-W(x(s)-D(x(s-\tau)))+\frac{\beta_{4}^{2}}{4 \beta_{1}}|x(s)-x(s-\tau)|^{2} \\
-\frac{\beta_{4}^{2}}{\beta_{1}\left(1-2 \kappa^{2}\right)} \int_{s-\tau}^{s}\left[\tau|f(x(v), x(v-\tau), r(v), v)|^{2}\right. \\
\left.+|g(x(v), x(v-\tau), r(v), v)|^{2}\right] d v .
\end{gathered}
$$

Substituting this into (3.10) implies

$$
\begin{aligned}
& \mathbb{E} V\left(\bar{x}_{t \wedge \sigma_{k}}, \bar{r}_{t \wedge \sigma_{k}}, t \wedge \sigma_{k}\right) \\
& \leq V\left(\bar{x}_{0}, \bar{r}_{0}, 0\right)+H_{1}-H_{2}+H_{3}-H_{4},
\end{aligned}
$$


where

$$
\begin{aligned}
& H_{1}=\mathbb{E} \int_{0}^{t \wedge \sigma_{k}}\left[-\alpha_{1} U_{1}(x(s), s)+\alpha_{2} U_{1}(x(s-\tau), s-\tau)\right] d s, \\
& H_{2}=\mathbb{E} \int_{0}^{t \wedge \sigma_{k}} W(x(s)-D(x(s-\tau))) d s \\
& H_{3}=\frac{\beta_{4}^{2}}{4 \beta_{1}} \mathbb{E} \int_{0}^{t \wedge \sigma_{k}}|x(s)-x(s-\tau)|^{2} d s, \\
& H_{4}=\frac{\beta_{4}^{2}}{\beta_{1}\left(1-2 \kappa^{2}\right)} \mathbb{E} \int_{0}^{t \wedge \sigma_{k}} \int_{s-\tau}^{s}\left[\tau|f(x(v), x(v-\tau), r(v), v)|^{2}\right. \\
& \left.\quad+|g(x(v), x(v-\tau), r(v), v)|^{2}\right] d v d s .
\end{aligned}
$$

Noting that

$$
\int_{0}^{t \wedge \sigma_{k}} U_{1}(x(s-\tau), s-\tau) d s \leq \int_{-\tau}^{t \wedge \sigma_{k}} U_{1}(x(v), v) d v
$$

we have

$$
H_{1} \leq \alpha_{2} \int_{-\tau}^{0} U_{1}(\eta(s), s) d s-\alpha_{3} \mathbb{E} \int_{0}^{t \wedge \sigma_{k}} U_{1}(x(s), s) d s,
$$

where $\alpha_{3}=\alpha_{1}-\alpha_{2}>0$ by condition (3.3). Substituting this into (3.11) yields

$$
\alpha_{3} \mathbb{E} \int_{0}^{t \wedge \sigma_{k}} U_{1}(x(s), s) d s \leq C_{1}-H_{2}+H_{3}-H_{4},
$$

where $C_{1}$ is a constant defined by

$$
C_{1}=V\left(\bar{x}_{0}, \bar{r}_{0}, 0\right)+\alpha_{2} \int_{-\tau}^{0} U_{1}(\eta(s), s) d s .
$$

Applying the classical Fatou lemma and let $k \rightarrow \infty$ in (3.12) to obtain

$$
\alpha_{3} \mathbb{E} \int_{0}^{t} U_{1}(x(s), s) d s \leq C_{1}-\bar{H}_{2}+\bar{H}_{3}-\bar{H}_{4},
$$

where

$$
\begin{aligned}
\bar{H}_{2}= & \mathbb{E} \int_{0}^{t} W(x(s)-D(x(s-\tau))) d s, \\
\bar{H}_{3}= & \frac{\beta_{4}^{2}}{4 \beta_{1}} \mathbb{E} \int_{0}^{t}|x(s)-x(s-\tau)|^{2} d s, \\
\bar{H}_{4}= & \frac{\beta_{4}^{2}}{\beta_{1}\left(1-2 \kappa^{2}\right)} \mathbb{E} \int_{0}^{t} \int_{s-\tau}^{s}\left[\tau|f(x(v), x(v-\tau), r(v), v)|^{2}\right. \\
& \left.\quad+|g(x(v), x(v-\tau), r(v), v)|^{2}\right] d v d s .
\end{aligned}
$$

Noting that $W \in C\left(\mathbb{R}^{n} ; \mathbb{R}_{+}\right)$, (3.13) implies

$$
\alpha_{3} \mathbb{E} \int_{0}^{t} U_{1}(x(s), s) d s \leq C_{1}+\bar{H}_{3}-\bar{H}_{4} .
$$

By the well-known Fubini theorem, we have

$$
\bar{H}_{3}=\frac{\beta_{4}^{2}}{4 \beta_{1}} \int_{0}^{t} \mathbb{E}|x(s)-x(s-\tau)|^{2} d s .
$$

For $t \in[0, \tau]$, we have

$$
\begin{aligned}
\bar{H}_{3} & \leq \frac{\beta_{4}^{2}}{2 \beta_{1}} \int_{0}^{\tau}\left(\mathbb{E}|x(s)|^{2}+\mathbb{E}|x(s-\tau)|^{2}\right) d s \\
& \leq \frac{\tau \beta_{4}^{2}}{\beta_{1}}\left(\sup _{-\tau \leq v \leq \tau} \mathbb{E}|x(v)|^{2}\right)=: C_{2},
\end{aligned}
$$

where, as usual, $=$ : means 'denoted by'. For $t>\tau$, we have

$$
\bar{H}_{3} \leq C_{2}+\frac{\beta_{4}^{2}}{4 \beta_{1}} \int_{\tau}^{t} \mathbb{E}|x(s)-x(s-\tau)|^{2} d s .
$$

Noting that

$$
\begin{aligned}
& |x(s)-x(s-\tau)| \leq \mid[x(s)-D(x(s-\tau))] \\
& -[x(s-\tau)-D(x(s-2 \tau))]|+| D(x(s-\tau))-D(x(s-2 \tau)) \mid \\
& \leq \kappa|x(s-\tau)-x(s-2 \tau)|+\mid \int_{s-\tau}^{s} f(x(u), x(u-\tau), r(u), u) d u \\
& \quad+\int_{s-\tau}^{s} g(x(u), x(u-\tau), r(u), u) d B(u) \mid .
\end{aligned}
$$

Therefore, we have

$$
\begin{aligned}
\mathbb{E} \mid x(s) & -\left.x(s-\tau)\right|^{2} \leq 2 \kappa^{2} \mathbb{E}|x(s-\tau)-x(s-2 \tau)|^{2} \\
& +2 \mathbb{E} \mid \int_{s-\tau}^{s} f(x(u), x(u-\tau), r(u), u) d u \\
& +\left.\int_{s-\tau}^{s} g(x(u), x(u-\tau), r(u), u) d B(u)\right|^{2} \\
& \leq 2 \kappa^{2} \mathbb{E}|x(s-\tau)-x(s-2 \tau)|^{2} \\
& +4 \mathbb{E} \int_{s-\tau}^{s}\left[\tau|f(x(u), x(u-\tau), r(u), u)|^{2}\right. \\
& \left.+|g(x(u), x(u-\tau), r(u), u)|^{2}\right] d u,
\end{aligned}
$$

which shows

$$
\begin{gathered}
\int_{\tau}^{t} \mathbb{E}|x(s)-x(s-\tau)|^{2} d s \leq 2 \kappa^{2} \int_{\tau}^{t} \mathbb{E}|x(s-\tau)-x(s-2 \tau)|^{2} d s \\
+4 \mathbb{E} \int_{\tau}^{t} \int_{s-\tau}^{s}\left[\tau|f(x(u), x(u-\tau), r(u), u)|^{2}\right. \\
\left.\quad+|g(x(u), x(u-\tau), r(u), u)|^{2}\right] d u d s \\
\leq 2 \kappa^{2} \int_{0}^{t} \mathbb{E}|x(s)-x(s-\tau)|^{2} d s \\
+4 \mathbb{E} \int_{\tau}^{t} \int_{s-\tau}^{s}\left[\tau|f(x(u), x(u-\tau), r(u), u)|^{2}\right. \\
\left.\quad+|g(x(u), x(u-\tau), r(u), u)|^{2}\right] d u d s .
\end{gathered}
$$

Noting that $0<\kappa<\frac{\sqrt{2}}{2}$, it follows that

$$
\begin{gathered}
\int_{\tau}^{t} \mathbb{E}|x(s)-x(s-\tau)|^{2} d s \leq \frac{2 \kappa^{2}}{1-2 \kappa^{2}} \int_{0}^{\tau} \mathbb{E}|x(s)-x(s-\tau)|^{2} d s \\
+\frac{4}{1-2 \kappa^{2}} \mathbb{E} \int_{\tau}^{t} \int_{s-\tau}^{s}\left[\tau|f(x(u), x(u-\tau), r(u), u)|^{2}\right. \\
\left.\quad+|g(x(u), x(u-\tau), r(u), u)|^{2}\right] d u d s .
\end{gathered}
$$

Hence

$$
\begin{aligned}
\bar{H}_{3} & \leq C_{2}+\frac{2 \kappa^{2} \tau \beta_{4}^{2}}{\left(1-2 \kappa^{2}\right) \beta_{1}} \sup _{-\tau \leq v \leq \tau} \mathbb{E}|x(v)|^{2}+\bar{H}_{4} \\
& =C_{3}+\bar{H}_{4}
\end{aligned}
$$

where $C_{3}=C_{2}+\frac{2 \kappa^{2} \tau \beta_{4}^{2}}{\left(1-2 \kappa^{2}\right) \beta_{1}} \sup _{-\tau \leq v \leq \tau} \mathbb{E}|x(v)|^{2}$. Substituting this into (3.14) yields $\alpha_{3} \mathbb{E} \int_{0}^{t} U_{1}(x(s), s) d s \leq C_{1}+C_{3}$. Letting $t \rightarrow \infty$ gives

$$
\mathbb{E} \int_{0}^{\infty} U_{1}(x(s), s) d s \leq \frac{1}{\alpha_{3}}\left(C_{1}+C_{3}\right) .
$$

Similarly, we see from (3.11) that

$$
\begin{gathered}
\mathbb{E} U\left(x\left(t \wedge \sigma_{k}\right)-D\left(x\left(t \wedge \sigma_{k}-\tau\right)\right), r\left(t \wedge \sigma_{k}\right), t \wedge \sigma_{k}\right) \\
\leq C_{1}-H_{2}+H_{3}-H_{4} .
\end{gathered}
$$

Letting $k \rightarrow \infty$ we get

$$
\mathbb{E} U(x(t)-D(x(t-\tau)), r(t), t) \leq C_{1}+C_{3}<\infty,
$$


which shows

$$
\sup _{0 \leq t<\infty} \mathbb{E} U(x(t)-D(x(t-\tau)), r(t), t)<\infty .
$$

Thus the proof is complete.

We have established delay-dependent stability criteria for highly nonlinear NSDDEs even though the condition given by (3.7) is sufficient rather than necessary. The following corollary gives a criterion on $H_{\infty}$-stability.

Corollary 3.5. Let the conditions of Theorem 3.4 hold. If there moreover exists a pair of positive constants $c$ and $p$ such that

$$
c|x|^{p} \leq U_{1}(x, t), \quad \forall(x, t) \in \mathbb{R}^{n} \times \mathbb{R}_{+},
$$

then for any given initial data (2.2), the solution of the NSDDE (2.1) satisfies

$$
\int_{0}^{\infty} \mathbb{E}|x(t)|^{p} d t<\infty
$$

That is, the NSDDE (2.1) is $H_{\infty}$-stable in $L^{p}$.

This corollary follows from Theorem 3.4 obviously. However, it does not follow from (3.19) that $\lim _{t \rightarrow \infty} \mathbb{E}|x(t)|^{p}=0$.

Theorem 3.6. Let the conditions of Corollary 3.5 hold. If, moreover,

$$
p \geq 2 \text { and }\left(p+q_{1}-1\right) \vee\left(p+2 q_{2}-2\right) \leq q,
$$

then the solution of the NSDDE (2.1) satisfies

$$
\lim _{t \rightarrow \infty} \mathbb{E}|x(t)|^{p}=0
$$

for any initial data (2.2). That is, the NSDDE (2.1) is asymptotically stable in $L^{p}$.

Proof: Again, fix the initial data (2.2) arbitrarily. For any $0 \leq t_{1}<$ $t_{2}<\infty$, by the Itô formula, we get

$$
\begin{aligned}
& \mathbb{E}\left|x\left(t_{2}\right)-D\left(x\left(t_{2}-\tau\right)\right)\right|^{p}-\mathbb{E}\left|x\left(t_{1}\right)-D\left(x\left(t_{1}-\tau\right)\right)\right|^{p} \\
& =\mathbb{E} \int_{t_{1}}^{t_{2}}\left(p|x(t)-D(x(t-\tau))|^{p-2}(x(t)-D(x(t-\tau)))^{T}\right. \\
& \quad \times f(x(t), x(t-\tau), r(t), t) \\
& +\frac{p}{2}|x(t)-D(x(t-\tau))|^{p-2}|g(x(t), x(t-\tau), r(t), t)|^{2} \\
& +\frac{p(p-2)}{2}|x(t)-D(x(t-\tau))|^{p-4} \mid(x(t)-D(x(t-\tau)))^{T} \\
& \left.\quad \times\left. g(x(t), x(t-\tau), r(t), t)\right|^{2}\right) d t .
\end{aligned}
$$

This implies

$$
\begin{aligned}
& |\mathbb{E}| x\left(t_{2}\right)-\left.D\left(x\left(t_{2}-\tau\right)\right)\right|^{p}-\mathbb{E}\left|x\left(t_{1}\right)-D\left(x\left(t_{1}-\tau\right)\right)\right|^{p} \mid \\
& \leq \mathbb{E} \int_{t_{1}}^{t_{2}}\left(p|x(t)-D(x(t-\tau))|^{p-1}|f(x(t), x(t-\tau), r(t), t)|\right. \\
& \left.+\frac{p(p-1)}{2}|x(t)-D(x(t-\tau))|^{p-2}|g(x(t), x(t-\tau), r(t), t)|^{2}\right) d t \\
& \leq \mathbb{E} \int_{t_{1}}^{t_{2}}\left(p K|x(t)-D(x(t-\tau))|^{p-1}\left[1+|x(t)|^{q_{1}}+|x(t-\tau)|^{q_{1}}\right]\right. \\
& \quad+\frac{3 p(p-1) K^{2}}{2}|x(t)-D(x(t-\tau))|^{p-2} \\
& \left.\quad \times\left[1+|x(t)|^{2 q_{2}}+|x(t-\tau)|^{2 q_{2}}\right]\right) d t .
\end{aligned}
$$

By inequalities

$$
\begin{gathered}
|x(t)-D(x(t-\tau))|^{p} \leq 2^{p-1}\left(|x(t)|^{p}+|D(x(t-\tau))|^{p}\right) \\
\leq 2^{p-1}\left(|x(t)|^{p}+\kappa^{p}|x(t-\tau)|^{p}\right) \\
|x(t)|^{p-1}|x(t-\tau)|^{q_{1}} \leq|x(t)|^{p+q_{1}-1}+|x(t-\tau)|^{p+q_{1}-1} \\
|x(t)|^{p-1} \leq 1+|x(t)|^{q} .
\end{gathered}
$$

We can obtain

$$
\begin{gathered}
|\mathbb{E}| x\left(t_{2}\right)-\left.D\left(x\left(t_{2}-\tau\right)\right)\right|^{p}-\mathbb{E}\left|x\left(t_{1}\right)-D\left(x\left(t_{1}-\tau\right)\right)\right|^{p} \mid \\
\leq C_{4}\left(t_{2}-t_{1}\right),
\end{gathered}
$$

where

$$
C_{4}=2^{p+1}\left[p K+3 p(p-1) K^{2} / 2\right]\left(1+\sup _{-\tau \leq t<\infty} \mathbb{E}|x(t)|^{q}\right)<\infty .
$$

Thus we have $\mathbb{E}|x(t)-D(x(t-\tau))|^{p}$ is uniformly continuous in $t$ on $\mathbb{R}_{+}$. By (3.19) we have

$$
\begin{aligned}
\int_{0}^{\infty} \mathbb{E} \mid & x(t)-\left.D(x(t-\tau))\right|^{p} d t \\
& \leq \int_{0}^{\infty} 2^{p-1} \mathbb{E}\left(|x(t)|^{p}+\kappa^{p}|x(t-\tau)|^{p}\right) d t \\
& \leq 2^{p-1}\left(1+\kappa^{p}\right) \int_{0}^{\infty} \mathbb{E}|x(t)|^{p} d t+2^{p-1} \kappa^{p}\|\eta\|<\infty,
\end{aligned}
$$

so we obtain $\lim _{t \rightarrow \infty} \mathbb{E}|x(t)-D(x(t-\tau))|^{p}=0$. Applying the inequalities (2.5) and

$$
(a+b)^{p} \leq(1+\epsilon)^{p-1}\left(a^{p}+\epsilon^{1-p} b^{p}\right), \forall a, b \geq 0, p \geq 1, \epsilon>0,
$$

we have

$$
\begin{aligned}
& \mathbb{E}|x(t)|^{p} \leq \mathbb{E}[|x(t)-D(x(t-\tau))|+|D(x(t-\tau))|]^{p} \\
& \quad \leq \mathbb{E}\left[(1+\epsilon)^{p-1}\left(|x(t)-D(x(t-\tau))|^{p}+\epsilon^{1-p} \kappa^{p}|x(t-\tau)|^{p}\right)\right] .
\end{aligned}
$$

Setting $\epsilon=\kappa /(1-\kappa)$, we have

$$
\mathbb{E}|x(t)|^{p} \leq\left(\frac{1}{1-\kappa}\right)^{p-1} \mathbb{E}|x(t)-D(x(t-\tau))|^{p}+\kappa \mathbb{E}|x(t-\tau)|^{p},
$$

letting $t \rightarrow \infty$, we have

$$
\lim _{t \rightarrow \infty} \sup \mathbb{E}|x(t)|^{p} \leq \kappa \lim _{t \rightarrow \infty} \sup \mathbb{E}|x(t)|^{p} \quad \text { a.s. }
$$

This, together with the Lemma 2.3, yields $\lim _{t \rightarrow \infty} \mathbb{E}|x(t)|^{p}=0$. Thus the proof is complete.

Remark 3.7. In order to obtain the assertion $\lim _{t \rightarrow \infty} \mathbb{E}|x(t)|^{p}=0$, some new mathematical techniques have been applied compared with [16]. In general it is not possible to imply $\lim _{t \rightarrow \infty} U_{1}(x(t), t)=$ 0 a.s. from (3.8) and hence get $\lim _{t \rightarrow \infty}|x(t)|=0$ a.s. To make this possible, we need some additional conditions as described in the following theorem. It should be pointed out that there is no such a result in [16].

Theorem 3.8. Let the conditions of Theorem 3.4 hold. If there moreover exists pair of positive constants $c$ and $p$ such that

$$
c|x|^{p} \leq U_{1}(x, t) \quad \text { and } \quad\left(q_{1}+1\right) \vee\left(2 q_{2}\right) \leq p .
$$

Then for any given initial date $\eta$ in (2.2), the solution obeys that

$$
\lim _{t \rightarrow \infty} x(t)=0 \quad \text { a.s. }
$$

Proof: Again fix the initial data $\eta$ in (2.2) arbitrarily. From (3.13) we can show that

$$
\int_{0}^{\infty} \mathbb{E} W(x(t)-D(x(t-\tau))) d t<\infty .
$$

By the Fubini theorem we have

$$
C_{5}:=\mathbb{E} \int_{0}^{\infty} W(x(t)-D(x(t-\tau))) d t<\infty,
$$

which implies

$$
\int_{0}^{\infty} W(x(t)-D(x(t-\tau))) d t<\infty \quad \text { a.s. }
$$


Setting $z(t)=x(t)-D(x(t-\tau))$ for $t \geq 0$ with $\sigma_{k}:=\inf \{t \geq$ $0:|z(t)|=k\}$. We observe from (3.23) that

$$
\lim _{t \rightarrow \infty} \inf W(z(t))=0 \quad \text { a.s. }
$$

By Corollary 3.5, we have $C_{6}:=\int_{0}^{\infty} \mathbb{E}|x(t)|^{p} d t<\infty$. Moreover, in the same way as Theorem 3.6 was proved, we can show that

$$
\begin{aligned}
\mathbb{E}\left|z\left(T \wedge \sigma_{k}\right)\right|^{p} & \leq C_{7}+C_{8} \int_{0}^{\infty} \mathbb{E}|x(t)|^{p} d t \\
& =C_{7}+C_{6} C_{8}:=C, \quad \forall T>0,
\end{aligned}
$$

where $C_{7}=2^{p-1} \kappa^{p}\|\eta\|, C_{8}=2^{p-1}\left(1+\kappa^{p}\right)$. This implies

$$
k^{p} \mathbb{P}\left(\sigma_{k} \leq T\right) \leq C .
$$

Letting $T \rightarrow \infty$ yields

$$
k^{p} \mathbb{P}\left(\sigma_{k}<\infty\right) \leq C .
$$

We now claim that

$$
\lim _{t \rightarrow \infty} W(z(t))=0 \quad \text { a.s. }
$$

In fact, if this is false, then we can find a number $\varepsilon \in(0,1 / 4)$ such that

$$
\mathbb{P}\left(\Omega_{1}\right) \geq 4 \varepsilon,
$$

where $\Omega_{1}=\left\{\lim _{t \rightarrow \infty} \sup W(z(t))>2 \varepsilon\right\}$. Recalling (3.25), we can find an integer $m$ sufficiently large for $\mathbb{P}\left(\sigma_{m}<\infty\right) \leq \varepsilon$. This means that

$$
P\left(\Omega_{2}\right) \geq 1-\varepsilon,
$$

where $\Omega_{2}:=\{|z(t)|<m$ for $\forall t \geq-\tau\}$. By (3.27) and (3.28) we get

$$
\mathbb{P}\left(\Omega_{1} \cap \Omega_{2}\right) \geq \mathbb{P}\left(\Omega_{1}\right)-\mathbb{P}\left(\Omega_{2}^{c}\right) \geq 3 \varepsilon,
$$

where $\Omega_{2}^{c}$ is the complement of $\Omega_{2}$. Let us now define the stopped process $\zeta(t)=z\left(t \wedge \sigma_{m}\right)$ for $t \geq-\tau$. Clearly, $\zeta(t)$ is a bounded Itô process with its differential

$$
d \zeta(t)=\phi(t) d t+\psi(t) d B(t)
$$

where

$$
\begin{aligned}
& \phi(t)=f(x(t), x(t-\tau), t, r(t)) I_{\left[0, \sigma_{m}\right)}(t), \\
& \psi(t)=g(x(t), x(t-\tau), t, r(t)) I_{\left[0, \sigma_{m}\right)}(t) .
\end{aligned}
$$

For $0 \leq t<\sigma_{m}$, by (2.5) we have

$|x(t)| \leq|x(t)-D(x(t-\tau))|+|D(x(t-\tau))| \leq m+\kappa|x(t-\tau)|$,

which shows

$$
\sup _{0 \leq t<\sigma_{m}}|x(t)| \leq m+\kappa\|\eta\|+\kappa \sup _{0 \leq t<\sigma_{m}}|x(t)| .
$$

Therefore, we have

$$
\sup _{-\tau \leq t<\sigma_{m}}|x(t)| \leq\left(\frac{1}{1-\kappa}(m+\kappa\|\eta\|)\right) \vee\|\eta\| .
$$

Recalling the polynomial growth condition (2.4), from (3.31) we see that $\phi(t)$ and $\psi(t)$ are bounded processes, say

$$
|\phi(t)| \vee|\psi(t)| \leq C_{9} \quad \text { a.s. }
$$

for all $t \geq 0$ and some $C_{9}>0$. Moreover, we also observe that $|\zeta(t)| \leq m$ for all $t \geq-\tau$. Define a sequence of stopping times

$$
\begin{gathered}
\rho_{1}=\inf \{t \geq 0: W(\zeta(t)) \geq 2 \varepsilon\}, \\
\rho_{2 j}=\inf \left\{t \geq \rho_{2 j-1}: W(\zeta(t)) \leq \varepsilon\right\}, \quad j=1,2, \cdots, \\
\rho_{2 j+1}=\inf \left\{t \geq \rho_{2 j}: W(\zeta(t)) \geq 2 \varepsilon\right\}, \quad j=1,2, \cdots .
\end{gathered}
$$

Note from (3.24) and the definition of $\Omega_{1}$ and $\Omega_{2}$, we have

$$
\Omega_{1} \cap \Omega_{2} \subset\left\{\sigma_{m}=\infty\right\} \bigcap\left(\cap_{j=1}^{\infty}\left\{\rho_{j}<\infty\right\}\right) .
$$

We also note that for all $\omega \in \Omega_{1} \cap \Omega_{2}$, and $j \geq 1$,

$$
\begin{gathered}
W\left(\zeta\left(\rho_{2 j-1}\right)\right)-W\left(\zeta\left(\rho_{2 j}\right)\right)=\varepsilon \quad \text { and } \\
W(\zeta(t)) \geq \varepsilon \quad \text { when } t \in\left[\rho_{2 j-1}, \rho_{2 j}\right] .
\end{gathered}
$$

Since $W(\cdot)$ is uniformly continuous in the close ball $\bar{S}_{m}=\{x \in$ $\left.\mathbb{R}^{n}:|x| \leq m\right\}$. We can choose $\delta=\delta(\varepsilon)>0$ small sufficiently for which

$$
\left|W\left(\zeta_{1}\right)-W\left(\zeta_{2}\right)\right|<\varepsilon, \zeta_{1}, \zeta_{2} \in \bar{S}_{m}, \text { with }\left|\zeta_{1}-\zeta_{2}\right|<\delta .
$$

We highlight that for $\omega \in \Omega_{1} \cap \Omega_{2}$, if $\left|\zeta\left(\rho_{2 j-1}+u\right)-\zeta\left(\rho_{2 j-1}\right)\right|<\delta$ for all $u \in[0, \lambda]$ and some $\lambda>0$, then $\rho_{2 j}-\rho_{2 j-1} \geq \lambda$. Choose a sufficiently small positive number $\lambda$ and then a sufficiently large positive integer $j_{0}$ such that

$$
2 C_{9}^{2} \lambda(\lambda+4) \leq \varepsilon \delta^{2} \text { and } C_{5}<\varepsilon^{2} \lambda j_{0} .
$$

By (3.29) and (3.33), we can further choose a sufficiently large number $T$ for

$$
\mathbb{P}\left(\rho_{2 j_{0}} \leq T\right) \geq 2 \varepsilon
$$

In particular, if $\rho_{2 j_{0}} \leq T$, then $\left|\zeta\left(\rho_{2 j_{0}}\right)\right|<m$, and hence $\rho_{2 j_{0}}<\sigma_{m}$ by the definition of $\zeta(t)$. We hence have

$$
\zeta(t, \omega)=z(t, \omega) \text { for all } 0 \leq t \leq \rho_{2 j_{0}} \text { and } \omega \in\left\{\rho_{2 j_{0}} \leq T\right\} .
$$

By the Hölder inequality and the Burkholder-Davis-Gundy inequality (see, e.g.,[4, Theorem 1.7 .3 on page 40$]$ ), we can have that, for $1 \leq$ $j \leq j_{0}$,

$$
\begin{aligned}
& \mathbb{E}\left(\sup _{0 \leq t \leq \lambda}\left|\zeta\left(\rho_{2 j-1} \wedge T+t\right)-\zeta\left(\rho_{2 j-1} \wedge T\right)\right|^{2}\right) \\
& \left.\quad \leq 2 \lambda \mathbb{E} \int_{\rho_{2 j-1} \wedge T}^{\rho_{2 j-1} \wedge T+\lambda}|\phi(s)|^{2} d s+8 \mathbb{E} \int_{\rho_{2 j-1} \wedge T}^{\rho_{2 j-1} \wedge T+\lambda}|\psi(s)|^{2} d s\right) \\
& \leq 2 C_{9}^{2} \lambda(\lambda+4) .
\end{aligned}
$$

This, together with (3.36) and Markov inequality, we can obtain that

$$
\mathbb{P}\left(\sup _{0 \leq t \leq \lambda}\left|\zeta\left(\rho_{2 j-1} \wedge T+t\right)-\zeta\left(\rho_{2 j-1} \wedge T\right)\right| \geq \delta\right) \leq \varepsilon .
$$

Noting that $\rho_{2 j-1} \leq T$ if $\rho_{2 j_{0}} \leq T$, we can derive from (3.37) and the above inequality that

$$
\begin{aligned}
& \mathbb{P}\left(\left\{\rho_{2 j_{0}} \leq T\right\} \cap\left\{\sup _{0 \leq t \leq \lambda}\left|\zeta\left(\rho_{2 j-1}+t\right)-\zeta\left(\rho_{2 j-1}\right)\right|<\delta\right\}\right) \\
& =\mathbb{P}\left(\rho_{2 j_{0}} \leq T\right) \\
& -\mathbb{P}\left(\left\{\rho_{2 j_{0}} \leq T\right\} \cap\left\{\sup _{0 \leq t \leq \lambda}\left|\zeta\left(\rho_{2 j-1}+t\right)-\zeta\left(\rho_{2 j-1}\right)\right| \geq \delta\right\}\right) \\
& \geq \mathbb{P}\left(\rho_{2 j_{0}} \leq T\right)-\mathbb{P}\left(\sup _{0 \leq t \leq \lambda}\left|\zeta\left(\rho_{2 j-1}+t\right)-\zeta\left(\rho_{2 j-1}\right)\right| \geq \delta\right) \geq \varepsilon .
\end{aligned}
$$

This, together with (3.35), implies easily that

$$
\mathbb{P}\left(\left\{\rho_{2 j_{0}} \leq T\right\} \cap\left\{\rho_{2 j}-\rho_{2 j-1} \geq \lambda\right\}\right) \geq \varepsilon .
$$

By (3.22), (3.38) and (3.39), we derive

$$
\begin{aligned}
C_{5} & \geq \sum_{j=1}^{j_{0}} \mathbb{E}\left(I_{\left\{\rho_{2 j_{0}} \leq T\right\}} \int_{\rho_{2 j-1}}^{\rho_{2 j}} W(z(t)) d t\right) \\
& \geq \varepsilon \sum_{j=1}^{j_{0}} \mathbb{E}\left(I_{\left\{\rho_{2 j_{0}} \leq T\right\}}\left(\rho_{2 j}-\rho_{2 j-1}\right)\right) \\
& \geq \varepsilon \lambda \sum_{j=1}^{j_{0}} \mathbb{P}\left(\left\{\rho_{2 j_{0}} \leq T\right\} \cap\left\{\rho_{2 j}-\rho_{2 j-1} \geq \lambda\right\}\right) \geq \varepsilon^{2} \lambda j_{0} .
\end{aligned}
$$


This contradicts the second inequality in (3.36). Thus (3.26) must hold.

We now claim $\lim _{t \rightarrow \infty} z(t)=0$ a.s. If this were not true, then $\varepsilon_{1}:=\mathbb{P}\left(\Omega_{3}\right)>0$, where $\Omega_{3}=\left\{\lim \sup _{t \rightarrow \infty}|z(t)|>0\right\}$. On the other hand, by (3.25), we can find a positive integer $m_{0}$ large enough for $\mathbb{P}\left(\sigma_{m_{0}}<\infty\right) \leq 0.5 \varepsilon_{1}$. Let $\Omega_{4}=\left\{\sigma_{m_{0}}=\infty\right\}$. Then

$$
\mathbb{P}\left(\Omega_{3} \cap \Omega_{4}\right) \geq \mathbb{P}\left(\Omega_{3}\right)-\mathbb{P}\left(\Omega_{4}^{c}\right) \geq 0.5 \varepsilon_{1} .
$$

For any $\omega \in \Omega_{3} \cap \Omega_{4}, z(t, \omega)$ is bounded on $t \in \mathbb{R}_{+}$. We can then find a sequence $\left\{t_{j}\right\}_{j \geq 1}$ such that $t_{j} \rightarrow \infty$ and $z\left(t_{j}, \omega\right) \rightarrow \bar{z}(\omega) \neq 0$ as $j \rightarrow \infty$. This, together with the continuity of $W$, implies

$$
\lim _{j \rightarrow \infty} W\left(z\left(t_{j}, \omega\right)\right)=W(\bar{z}(\omega))>0 .
$$

Consequently, we have

$$
\limsup _{t \rightarrow \infty} W(z(t, \omega))>0 \text { for all } \omega \in \Omega_{3} \cap \Omega_{4} .
$$

But this contradicts (3.26). We therefore must have the assertion $\lim _{t \rightarrow \infty} z(t)=0$ a.s. Hence we obtain

$$
\sup _{0 \leq t<\infty}|z(t)|<\infty \quad \text { a.s. }
$$

Finally, let us show our assertion (3.21). By (2.5), we have

$$
\begin{aligned}
|x(t)| & \leq|x(t)-D(x-\tau)|+|D(x-\tau)| \\
& \leq|z(t)|+\kappa|x(t-\tau)| \quad \text { a.s. }
\end{aligned}
$$

This implies, for any $T>0$,

$$
\sup _{0 \leq t \leq T}|x(t)| \leq \sup _{0 \leq t \leq T}|z(t)|+\kappa\|\eta\|+\kappa \sup _{0 \leq t \leq T}|x(t)| \quad \text { a.s. }
$$

Hence we deduce

$$
\sup _{0 \leq t \leq T}|x(t)| \leq \frac{1}{1-\kappa}\left(\sup _{0 \leq t \leq T}|z(t)|+\kappa\|\eta\|\right) \quad \text { a.s. }
$$

Letting $T \rightarrow \infty$ and using (3.40) we obtain that

$$
\sup _{0 \leq t<\infty}|x(t)|<\infty \quad \text { a.s. }
$$

By (3.41), letting $t \rightarrow \infty$, using $\lim _{t \rightarrow \infty} z(t)=0$ a.s., we obtain that

$$
\limsup _{t \rightarrow \infty}|x(t)| \leq \kappa \limsup _{t \rightarrow \infty}|x(t)| \quad \text { a.s. }
$$

For $\kappa \in\left(0, \frac{\sqrt{2}}{2}\right)$, together with (3.42), we must have

$$
\lim _{t \rightarrow \infty}|x(t)|=0 \quad \text { a.s. }
$$

which is the required assertion (3.21). The proof is complete. $\square$

Remark 3.9. Although the adopted methods and skills borrow from [16], the existence of the neutral term $D(x(t-\tau))$ essentially changes the problem, and a significant amount of new mathematics has been developed to deal with the difficulties due to the neutral term.

\section{CONCLUSiON}

Stability of NSDDEs have been studied for many years, most of the results in this area require that the coefficients of equations are linear or nonlinear but bounded by linear functions. In this paper, without the linear growth condition, we have established delay-dependent stability criteria for highly nonlinear NSDDEs by the method of Lyapunov function. The $H_{\infty}$ stability in $L^{p}$, asymptotic stability in $L^{p}$ and almost surely asymptotic stability are discussed in this paper. Although the condition imposed in (3.6) covers many NSDDEs, this condition may exclude some highly nonlinear hybrid NSDDEs, in our future work we will remove this restrictive condition and give a generalised result to include a much wider class of hybrid NSDDEs.

\section{ACKNOWLEDGEMENTS}

The authors would like to thank the editor and reviewers for their very helpful comments and suggestions. This research was supported in part by the Natural Science Foundation of China (No. 71571001), the Promoting Plan of Higher Education of Anhui Province (TSKJ2016B11, TSKJ2015B24), the Royal Society (WM160014, Royal Society Wolfson Research Merit Award), the Royal Society and the Newton Fund (NA160317, Royal Society-Newton Advanced Fellowship) and the EPSRC (EP/K503174/1).

\section{REFERENCES}

[1] W. Chen, W. Zheng, Y. Shen, Delay-dependent stochastic stability and $H_{\infty}$-control of uncertain neutral stochastic systems with time delay, IEEE. T. Automat. Contr. 54(2009)1660-1667.

[2] M. Frederic, Stability analysis of time-varying neutral timedelay systems, IEEE. T. Automat. Contr. 60(2016)540-546.

[3] V. B. Kolmanovskii, A. D. Myshkis, Applied theory of functional differential equations, Kluwer Academic Pub., Amsterdam, 1992.

[4] X. Mao, Stochastic differential equations and their applications, 2nd Edition, Horwood Pub., Chichester, 2007.

[5] X. Mao, C. Yuan, Stochastic differential equations with markovian switching, Imperial College Press, London, 2006.

[6] H. Chen, P. Shi, C. Lim, P. Hu, Exponential stability for neutral stochastic Markov systems with time-varying delay and its applications, IEEE. T. Cybernetics. 46(2016)1350-1362.

[7] Q. Luo, X. Mao, Y. Shen, New criteria on exponential stability of neutral stochastic differential delay equations, Syst. Control. Lett. 55(2006)826-834.

[8] X. Mao, Y. Shen, C. Yuan, Almost surely asymptotic stability of neutral stochastic differential delay equations with Markovian switching, Stoch. Proc. Appl. 118(2008)1385-1406.

[9] X. Mao, Exponential stability in mean square of neutral stochastic differential equations, Syst. Control. Lett. 26(1995)245-251.

[10] M. Milö̈ević, M. Jovanović, A Taylor polynomial approach in approximations of solution to pantograph stochastic differential equations with Markovian switching, Math. Comput. Model. 53(2011)280-293.

[11] R. Yang, H. Gao, J. Lam, P. Shi, New stability criteria for neural networks with distributed and probabilistic delays, Circ. Syst. Signal. Pr. 28(2009)505-522.

[12] C. Yuan, Z. Hou, J. Bao, Stability in distribution of neutral stochastic differential delay equations with Markovian switching, Stat. Probabil. Lett. 79(2009)1663-1673.

[13] L. Hu, X. Mao, Y. Shen, Stability and boundedness of nonlinear hybrid stochastic differential delay equations, Syst. Control. Lett. 62(2013)178-187.

[14] L. Hu, X. Mao, L. Zhang, Robust stability and boundedness of nonlinear hybrid stochastic differential delay equations, IEEE. T. Automat. Contr. 59(2013)2319-2332.

[15] M. Obradović, M. Milos̈ević, Stability of a class of neutral stochastic differential equations with unbounded delay and Markovian switching and the Euler-Maruyama method, J. Comput. Appl. Math. 309(2017)244-266.

[16] W. Fei, L. Hu, X. Mao, M. Shen, Delay dependent stability of highly nonlinear hybrid stochastic systems, Automatica. 82(2017)165-170.

[17] X. Mao, M. J. Rassias, Khasminskii-type theorems for stochastic differential delay equations, Stoch. Anal. Appl. 23(2005)10451069. 